\title{
Topological Imbert-Fedorov shift in Weyl semimetals
}

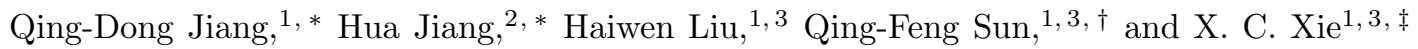 \\ ${ }^{1}$ International Center for Quantum Materials, School of Physics, Peking University, Beijing 100871, P.R. China \\ ${ }^{2}$ College of Physics, Optoelectronics and Energy, \\ Soochow University, Suzhou 215006, P.R. China \\ ${ }^{3}$ Collaborative Innovation Center of Quantum Matter, Beijing 100871, P.R. China
}

\begin{abstract}
The Goos-Hänchen (GH) shift and the Imbert-Fedorov (IF) shift are optical phenomena which describe the longitudinal and transverse lateral shifts at the reflection interface, respectively. Here, we report the GH and IF shifts in Weyl semimetals (WSMs) - a promising material harboring low energy Weyl fermions, a massless fermionic cousin of photons. Our results show that GH shift in WSMs is valley-independent which is analogous to that discovered in a $2 \mathrm{D}$ relativistic materialgraphene. However, the IF shift has never been explored in non-optical systems, and here we show that it is valley-dependent. Furthermore, we find that the IF shift actually originates from the topological effect of the system. Experimentally, the topological IF shift can be utilized to characterize the Weyl semimetals, design valleytronic devices of high efficiency, and measure the Berry curvature.
\end{abstract}

Introduction.-Modern quantum physics originates from understanding the wave-particle duality of all particles, among which photon is the first one being discovered with such duality. Classically, the physics of a beam of light being reflected at an interface is governed by geometric optics law, where the photons are treated as classical particles. In contrast, when considering the wave nature of photons, spatial shifts at the interface appear as longitudinal shift in the incident plane ${ }^{1 / 3}$, or transverse shift normal to the incident plane ${ }^{4} \sqrt{9}$, which are known as the Goos-Hänchen (GH) effect and the ImbertFedorov (IF) effect, respectively. Due to all particles possessing the wave-particle duality, the spatial shifts are also expected for other particles. For example, GH effect has been shown to exist in the systems of electrons 10 12, neutrons ${ }^{13}$, atoms $\frac{14}{14}$, etc. Particularly for the 2D massless Dirac fermions in graphene systems, the GH shift can be manipulated from positive to negative by tuning an external electric field ${ }^{15}$. However, the IF effect has not been studied in non-optical systems.

Similar to photons, Weyl particles are also massless. But different from photons, Weyl particles are spin $1 / 2$ chiral fermions and described by the Weyl equation. Recently, Weyl semimetals (WSMs) have been proposed as promising systems embedding Weyl fermions, generating intensive interests 16 22. In WSMs, the Weyl nodes always exist in pairs with opposite chiralities, and each Weyl node corresponds to a valley index ${ }^{23124}$. Several candidates are suggested to be WSMs including pyrochlore irradiates ${ }^{16}$, topological insulator and normal insulator heterostructures $\frac{18}{18}$, staggered flux states in cold atom systems 20 , and photonic crystals based on doublegyroid structures 21 . Despite of various theoretical prediction of WSMs, the experimental realization of WSMs remain a challenge. This failure cannot be ascribed to the impediment of material growth technique ${ }^{25 / 26}$, but to the scarcity of the method of direct experimental identification of WSMs 5 . Due to the topological properties of the Weyl fermions, the WSMs may possess exotic wave-packet dynamics, which indicates a new route to characterizing WSMs and potential applications in valleytronics 2831 .

In this paper, we report the GH effect and IF effect for 3D Weyl fermions in WSMs. By using wave packet method, we derive analytic results for the spatial shift of the GH effect and IF effect. Our results show that the GH shift is valley-independent. By contrast, the IF shift is valley-dependent[see Fig. 1], which give rise to the valley-dependent anomalous velocities in the system. Due to the IF shift being perpendicular to the incident plane, it is a generic $3 \mathrm{D}$ effect and could never appear in a $2 \mathrm{D}$ material, e.g. the graphene $\mathrm{e}^{15}$. Furthermore, we demonstrate that the IF shift originates from the topological effect of the system, namely, the Berry curvature of the system. Remarkably, the consequence of the valley-dependent anomalous velocity is significant enough to be detected experimentally. Finally, we discuss three applications of the valley-dependent IF shift: (i) effectively characterizing the WSMs; (ii) directly detecting the Berry curvature; (iii) efficiently inducing valley current with a high polarization rate.

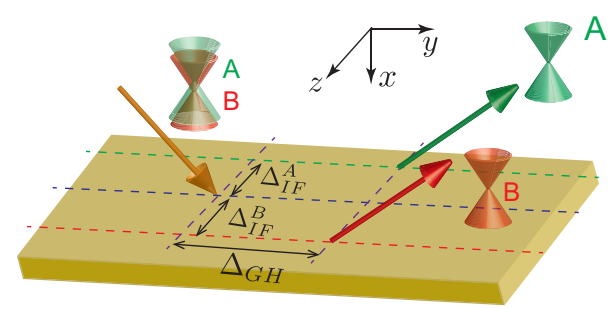

FIG. 1: Illustration of GH effect and IF effect in WSMs. The orange arrow represents the incident wave packet which include Weyl fermions from two valleys, whereas the green (red) arrow represents the reflected wave packet of Weyl fermions only from Valley A (B). $\Delta_{G H}$ denotes the GH shift, and $\Delta_{I F}^{A(B)}$ stands for the IF shift of valley A (B). This figure only shows the positive GH shift case.

Quantum GH and IF effects in WSMs.-The Hamil- 
tonian of the WSMs system is

$$
\mathcal{H}=\left\{\begin{array}{ll}
\sum_{i=x, y, z} v_{i} \hat{p}_{i} \sigma_{i} & (x \leqslant 0) \\
\sum_{i=x, y, z} v_{i}^{\prime} \hat{p}_{i} \sigma_{i}+V(x) & (x>0)
\end{array},\right.
$$

where $v_{i}\left(v_{i}^{\prime}\right)$ is velocity parameter for region $x \leqslant 0$ $(x>0) ; \hat{p}_{i}$ are momentum operators, $V(x)$ is potential, and $\sigma_{i}$ stand for Pauli matrices. To guarantee the model Hamiltonian Hermitian, we let $v_{x}=v_{x}^{\prime}$ in this paper. This Hamiltonian indicates an interface located at $x=0$ in the WSMs[see Fig. 2(a)]. We consider a beam of Weyl fermions incident from the region $x<0$ modeled by a Gaussian wave packet as $\psi_{g}^{\text {in }}(\mathbf{r})=\int_{-\infty}^{\infty} \int_{-\infty}^{\infty} d k_{y} d k_{z} f\left(k_{y}-\bar{k}_{y}\right) f\left(k_{z}-\bar{k}_{z}\right) \psi^{i n}(\mathbf{k}, \mathbf{r})$, where $f\left(k_{s}-\bar{k}_{s}\right)=\left(\sqrt{2 \pi} \Delta_{k_{s}}\right)^{-1} e^{-\left(k_{s}-\bar{k}_{s}\right)^{2} / 2 \Delta_{k_{s}}^{2}}$ are Gaussian distribution functions of width $\Delta_{k_{s}}$ peaked at the mean wave vector $\left(\bar{k}_{x}, \bar{k}_{y}, \bar{k}_{z}\right)$ with $s=y, z$. Note that none of our results depend on the shape of the wave packet. Here, $\psi^{i n}(\mathbf{k}, \mathbf{r})$ is the incident wave function, which is a solution of Weyl equation, i.e., $\mathcal{H} \psi^{i n}=E \psi^{i n}$ for region $x<0$ :

$$
\psi^{i n}(\mathbf{k}, \mathbf{r})=\frac{1}{\sqrt{1+\eta^{2}}}\left(\begin{array}{c}
e^{-i \alpha / 2} \\
\eta e^{i \alpha / 2}
\end{array}\right) e^{i k_{x} x+i k_{y} y+i k_{z} z},
$$

where $k_{x}=\sqrt{E^{2}-\left(\hbar v_{y} k_{y}\right)^{2}-\left(\hbar v_{z} k_{z}\right)^{2}} / \hbar v_{x}, \quad \alpha=$

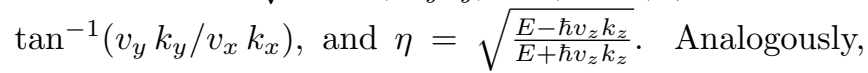
the reflected wave packet can be written as $\psi_{g}^{r e}(\mathbf{r})=$ $\int_{-\infty}^{\infty} \int_{-\infty}^{\infty} d k_{y} d k_{z} f\left(k_{y}-\bar{k}_{y}\right) f\left(k_{z}-\bar{k}_{z}\right) \psi^{r e}(\mathbf{k}, \mathbf{r})$, where $\psi^{r e}$ is the reflected wave function. $\psi^{r e}$ can be obtained from the incident wave Eq. (2) by the substitution $k_{x} \mapsto-k_{x}, \alpha \mapsto \pi-\alpha$ and multiplication with the reflection amplitude $r=|r| e^{i \phi_{r}}$. The integrals of $\psi_{g}^{i n}$ and $\psi_{g}^{r e}$ give the center of the wave packets, and therefore we can obtain the spatial shifts in $y, z$ directions ${ }^{32}$ :

$$
\left\{\begin{array}{c}
\Delta_{ \pm}^{y}=-\frac{\partial}{\partial k_{y}} \phi_{r}\left(\bar{k}_{y}, \bar{k}_{z}\right) \mp \frac{\partial}{\partial k_{y}} \alpha\left(\bar{k}_{y}, \bar{k}_{z}\right) \\
\Delta_{ \pm}^{z}=-\frac{\partial}{\partial k_{z}} \phi_{r}\left(\bar{k}_{y}, \bar{k}_{z}\right) \mp \frac{\partial}{\partial k_{z}} \alpha\left(\bar{k}_{y}, \bar{k}_{z}\right)
\end{array} .\right.
$$

The spatial shifts for Weyl fermions are defined as the average shifts of the two spinor components: $\Delta^{y(z)}=$ $\left(\Delta_{+}^{y(z)}+\eta^{2} \Delta_{-}^{y(z)}\right) /\left(1+\eta^{2}\right)$. In Eq.(3), $\alpha\left(\bar{k}_{y}, \bar{k}_{z}\right)$ represents the incident angle, and $\phi_{r}$ is the phase of the reflection coefficient, which can be obtained by matching the wave function at $x=0$. If the incident wave packet is confined in $x$-y plane, the in-plane shift $\Delta^{y}$ and out-of-plane shift $\Delta^{z}$ of wave packet corresponds to the GH shift $\Delta_{G H}$ and IF shift $\Delta_{I F}$ :

$$
\begin{aligned}
\Delta_{G H} & =\frac{v_{y}^{\prime}}{v_{x}^{\prime}} \frac{\left(1+\beta \sin ^{2} \bar{\alpha}-\frac{V}{E}\right)}{\beta \sin \bar{\alpha} \cos \bar{\alpha} \kappa} \\
\Delta_{I F} & =-C\left|\frac{\hbar v_{x} v_{z}}{v_{y}}\right| \frac{1}{E \tan \theta}\left[\frac{1-\frac{E}{V}(1-\gamma)}{1-\frac{E}{V}(1-\beta)}\right],
\end{aligned}
$$

where $\kappa=\sqrt{\left(\hbar v_{y}^{\prime} k_{y}\right)^{2}+\left(\hbar v_{z}^{\prime} k_{z}\right)^{2}-(E-V)^{2}} / \hbar v_{x}^{\prime}, \beta=$ $v_{y}^{\prime} / v_{y}, \gamma=v_{z}^{\prime} / v_{z}, \bar{\alpha}=\tan ^{-1}\left(v_{y} \bar{k}_{y} / v_{x} \bar{k}_{x}\right)$, and $\theta=$ $\tan ^{-1}\left(\bar{k}_{y} / \bar{k}_{x}\right) . C \equiv \operatorname{sgn}\left[v_{x} v_{y} v_{z}\right]$ is the chirality of the valley in WSMs.
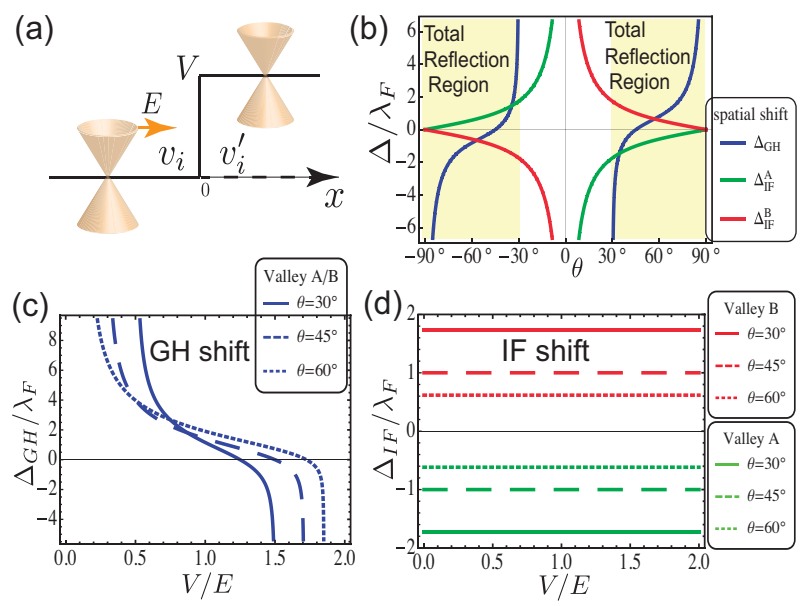

FIG. 2: (a) Schematic of p-n junction with an interface at $x=$ 0. $v_{i}\left(v_{i}^{\prime}\right)$ is the velocity in the left (right) side of the interface. $E$ is the Fermi energy, and $V$ is the potential difference of the junction. (b) GH shift $\Delta_{G H}$ and IF shift $\Delta_{I F}^{A(B)}$ of valley A (B) versus incident angle $\theta$. Total reflection happens at $\theta \geqslant 30^{\circ}$ (the yellow color shadow region). $\lambda_{F}=h v_{i} / E$ stands for the wave length of Weyl fermions. (c) Potential dependence of the GH shift for incident angle $\theta=30^{\circ}$ (solid), $45^{\circ}$ (dashed), and $60^{\circ}$ (dotted). (d) Potential dependence of the IF shift for incident angle $\theta=30^{\circ}$ (solid), $45^{\circ}$ (dashed), and $60^{\circ}$ (dotted).

We take the incident Fermi energy $E=100 \mathrm{meV}$, potential $V=150 \mathrm{meV}$, and velocities $v_{i}=v_{i}^{\prime}=10^{6} \mathrm{~m} / \mathrm{s}$, where $i=x, y, z$. In this case, $\beta=\gamma=1$. Note that the two valleys (A/B) of a WSMs have opposite chiralities. Fig. 2(b) shows the spatial shifts versus the incident angle $\theta$, where both the GH and IF shifts are odd functions of incident angle, which is consistent with symmetry analysis. Fig. 2(c) shows the valley independence and potential dependence of GH shift $\Delta_{G H}$, which can be tuned from positive to negative by external field $V$. This feature is analogous to the GH shift in graphene $e^{15}$. Fig. 2(d) illustrates that the IF shift is independent of potential, but depends on valley index. The IF shift can be utilized to manipulate the valley degree of freedom.

Topological origin of the IF effect. - Based on the semiclassical dynamics of wave packet, we show that the IF shift is closely related to the Berry curvature of the system. Let us assume the velocity $v_{i}=v_{i}^{\prime}(i=x, y, z)$ in WSMs system. In this case, $\beta=\gamma=1$, and Eq.(5) reduces to $\Delta_{I F}=-\left(\frac{\hbar v_{x} v_{z}}{v_{y}}\right) \frac{1}{E \tan \theta}$. Commonly, the Weyl node can be regarded as a magnetic monopole in $k$-space ${ }^{33}$, and thereby generates an effective magnetic field in $k$-space. The Berry curvature of Hamiltonian Eq. (1) in region $(x \leq 0)$ is $\boldsymbol{\Omega}^{ \pm}=\mp \frac{\hbar^{3} v_{x} v_{y} v_{z} \mathbf{k}}{2 E^{3}}$ for conduction band and valence band ${ }^{1}$, respectively. The semi- 


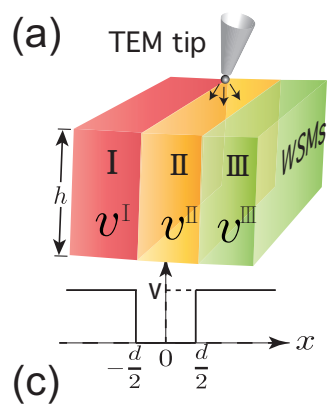

(b)
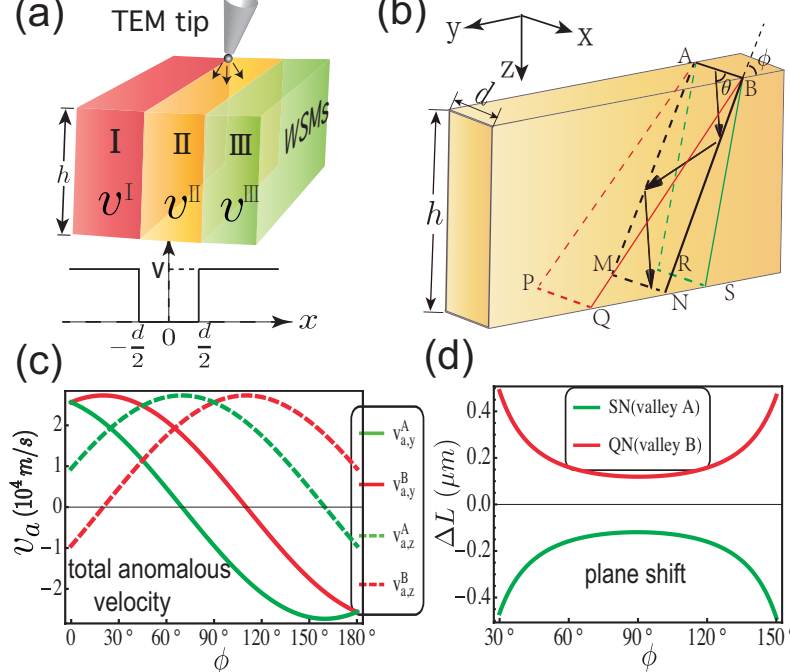

(d)

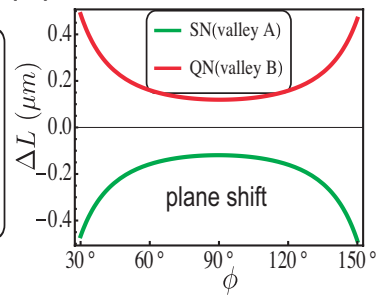

FIG. 3: (a) Schematic of the valley splitter for Weyl fermions. Region I, II, and III are three WSM layers with different velocities $\mathbf{v}^{I}, \mathbf{v}^{I I}$, and $\mathbf{v}^{I I}$. (b) Illustration of wave packet trajectory (black arrow) of Weyl fermions in region II. The electrons are injected by TEM tip with incident polar angle $\theta$ and azimuthal angle $\phi$. The parameter $\theta$ is fixed $50^{\circ}$ in our calculation. In ordinary case, the trajectory of wave packet stays in ABMN plane. However, due to the valley-dependent IF shift, the trajectory of Weyl fermions from valley A (B) would shift to plane ABRS (ABPQ). (c) $\phi$ dependence of the total anomalous velocities. (d) The propagating plane shift for two valleys. After considering IF shift, SN (QN) is the final position shift for valley A (B).

classical equation of motion $(\mathrm{EOM})^{1135}$ of wave packet is $\frac{d \mathbf{r}}{d t}=\frac{\partial E(\mathbf{k})}{\hbar \partial \mathbf{k}}-\frac{d \mathbf{k}}{d t} \times \boldsymbol{\Omega}$, where $\mathbf{r}$ and $\mathbf{k}$ are the center positions of the wave packet in phase space. We assume that the incident wave packet locates in the conduction band, and consider the incident wave packet in x-y plane with $k_{z}=0$. The variation of $k_{x}$ by potential $\mathrm{V}$ and nonzero Berry curvature $\Omega_{y}$ leads to the IF shift $\Delta_{I F}$ in $z$-direction:

$$
\Delta_{I F}=-\int_{k_{x}}^{-k_{x}} d k_{x} \Omega_{y}=-\left(\frac{\hbar v_{x} v_{z}}{v_{y}}\right) \frac{1}{E \tan \theta},
$$

where $\tan \theta=k_{y} / k_{x}$. Remarkably, Eq.(6) and Eq.(5) completely coincide in the case of $\beta=\gamma$. This coincidence does not depend on linear dispersion of the system $^{32}$. The consistency between Berry curvature calculations and wave packet results strongly support that the IF shift is mainly a topological effect. The IF shift in WSMs is quite different from that in optical systems. Particularly, $\Delta_{I F}$ reaches the maximum in WSMs [see Fig. 2(b)] comparing the zero value in optical systems at $\theta=0$ [6] This is because the conservation of $k_{y}, k_{z}$ guarantee the Weyl Fermions stay in the same valley during the reflection processes in WSMs. In contrast, the polarization of the photons change during the reflection processes, which will severely influence $\Delta_{I F}$. However,the semiclassical equation cannot be generally applied to all cases of reflection process because of the breakdown of adiabatic approximation for some systems.

Anomalous velocities induced by IF effect.-We consider a well collimated beam of Weyl fermions propagating in the middle layer (Region II) of a sandwich structure, which is constructed by three layers of WSMs[see Fig. 3(a)]. The applied electric potential profile is shown below the sandwich structure. The height of the structure is $h$ and the width of the region II is $d$. There are both GH and IF shifts at the two interfaces $\left(x= \pm \frac{d}{2}\right)$. In order to observe the valley-dependent IF shift, the mirror symmetry about $x=0$ plane need to be broken ${ }^{32}$. Thus, we consider that the $\mathrm{z}$ direction velocities in the three regions are different, with $v_{z}^{\mathrm{I}}=v_{L}$, $v_{z}^{\mathrm{II}}=v$, and $v_{z}^{\mathrm{III}}=v_{R}$; whereas the $\mathrm{x}$ and $\mathrm{y}$ directions velocities are still identical $v_{x / y}^{\mathrm{I}}=v_{x / y}^{\mathrm{II}}=v_{x / y}^{\mathrm{III}}=v$. Without considering the spatial shifts at the interfaces, the normal velocities in region II in $y$ and $z$ directions are $v_{n, y}=v \sin \theta \cos \phi$ and $v_{n, z}=v \sin \theta \sin \phi$, where angles $\theta$ and $\phi$ characterize the incident direction of the wave packet [see Fig. 3(b)]. We denote the GH and IF shifts at the left (right) interface as $\Delta_{G H}^{L(R)}$ and $\Delta_{I F}^{L(R)}$, respectively. During multiple reflections in region II, the GH and IF shifts are accumulated, and induce average anomalous velocities: $v_{a, y}=$ $\left[\left(\Delta_{G H}^{L}+\Delta_{G H}^{R}\right) \cos \phi+\left(\Delta_{I F}^{L}+\Delta_{I F}^{R}\right) \sin \phi\right] /(2 \Delta t)$ and $v_{a, z}=\left[\left(\Delta_{G H}^{L}+\Delta_{G H}^{R}\right) \sin \phi+\left(\Delta_{I F}^{L}+\Delta_{I F}^{R}\right) \cos \phi\right] /(2 \Delta t)$. $\Delta t=d /(v \cos \theta)$ represents the propagating time between two subsequent reflections. Therefore, the normal and anomalous velocity result in the total velocity: $v_{t, y(z)}=v_{n, y(z)}+v_{a, y(z)}$.

We set the parameters $E=100 \mathrm{meV}, V=150 \mathrm{meV}$, $h=10 \mu \mathrm{m}, d=50 \mathrm{~nm}, v_{z}^{I}=v_{L}=1.2 \times 10^{6} \mathrm{~m} / \mathrm{s}$, $v_{z}^{I I}=v=10^{6} \mathrm{~m} / \mathrm{s}, v_{z}^{I I I}=v_{R}=0.8 \times 10^{6} \mathrm{~m} / \mathrm{s}$, and velocities in other directions are all set as $10^{6} \mathrm{~m} / \mathrm{s}^{36}$. Fig. 3(c) shows $\phi$ dependence of the total anomalous velocities induced by the spatial shifts. The anomalous velocities are valley-dependent, which implies that the total velocities also depend on valley index. Eventually, the different velocities of the two valleys lead to macroscopic separation in real space, which is experimentally detectable. Fig. 3(d) shows the opposite position shift at the bottom of region II for valley A and valley $\mathrm{B}$. GH shift cannot induce the position shift, because GH shift always lie in the propagating plane. In contrast, IF shift can induce the position shifts ( $\mu \mathrm{m}$ order) for two valleys $(\mathbf{S N} \text { and } \mathbf{Q N})^{32}$.

Identification of WSMs. - The experimental verification of WSMs has not been justified mainly due to the scant of efficient detection method 38 40. The direct ARPES measurements of energy dispersions are currently scant due to the constraint of magnetic properties of WSMs. Here, we suggest that the IF effect can be used as an experimental identification of WSMs. It has been shown that the topological IF shift splits the incident wave packets into opposite directions respect to the 

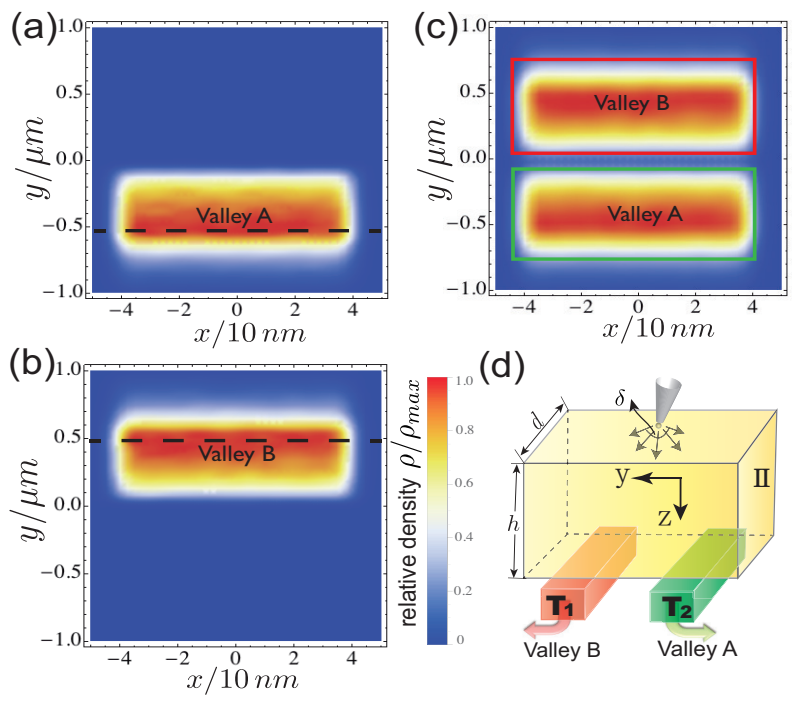

(d)

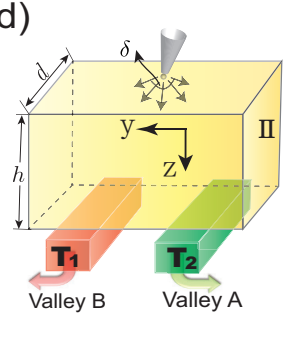

FIG. 4: Valley density distribution shift caused by IF effect. (a), (b) and (c) show the relative density distribution of valley $\mathrm{A}$, valley $\mathrm{B}$, and both, respectively, after considering $\mathrm{GH}$ and IF effect. The color bar on the right represents the relative density $\rho / \rho_{\max }$ of Weyl fermions. Here, $\rho\left(\rho_{\max }\right)$ represents the absolute (maximum) density of Weyl fermions at the bottom. The dashed lines indicate the location of the maximum intensity of density. Red (Green) box in Fig. (c) guides eyes to the region where only valley $\mathrm{B}(\mathrm{A})$ exist (pure valley polarization). (d) Proposed setup for generating valley current. The devices $\mathbf{T}_{1}$ (red) and $\mathbf{T}_{2}$ (green) are two terminals, which can extracted valley current out.

valley index. Thus, the splitting of wave packets on the bottom of region II in Fig. 3(a) can serve as a hallmark of WSMs. Furthermore, even considering an incident wave packet with finite angle range, this exotic splitting can also exist.

Pure valley polarization. - Let us consider an TEM injector with incident angle range $\delta\left(\theta \in\left[\theta_{c}-\delta / 2, \theta_{c}+\delta / 2\right]\right.$, and $\left.\phi \in\left[\phi_{c}-\delta / 2, \phi_{c}+\delta / 2\right]\right)$ at the top of Region II, and study the valley density distribution at the bottom [see Fig. 4(d)]. We calculate the valley density distribution with parameters $E=100 \mathrm{meV}, V=150 \mathrm{meV}$, $h=10 \mu \mathrm{m}, d=80 \mathrm{~nm}, \delta=3^{\circ}, \theta_{c}=32.5^{\circ}$, and $\phi_{c}=90^{\circ}$. Without considering anomalous velocities, the density distributions for valley $\mathrm{A}$ and $\mathrm{B}$ are maximized at the center of y direction, and thus not distinguishable ${ }^{32}$. In contrast, after considering the anomalous velocities, Fig. 4(a), (b) and (c) show the density distributions for valley $A$, valley $B$, and valley $A \& B$, respectively. The IF effect induces opposite shifts in y-direction for valley $\mathrm{A}$ and valley B. Since the Weyl fermions from valleys A and B are well separated in space of micrometer order, pure valley current can be generated in the green (red) regions[see Fig. 4(c)]. This schematic set-up in Fig. 4(d) can be utilized to generate pure valley current.

Detection of Berry curvature. - The Berry curvaturea gauge invariant quantity - should be detectable in experiment ${ }^{1}$. However, up to date, there lacks an experimental feasible method to measure the Berry curvature in real materials. The topological IF effect provides a new way to measure the Berry curvature. For a system with inversion symmetry, the Berry curvature is an even function of wave vector $\mathbf{k}$, i.e., $\Omega(\mathbf{k})=\boldsymbol{\Omega}(-\mathbf{k})^{1}$, which is usually satisfied in WSM $\$ 16$. Considering a wave packet propagating in $x-y$ plane with $k_{z}=0$, the IF shift can be expressed as $\Delta_{I F}=2 \int_{0}^{k_{x}} d k_{x} \Omega_{y}(\mathbf{k})$, or

$$
\Omega_{y}(\mathbf{k})=\frac{1}{2} \frac{\partial \Delta_{I F}(\mathbf{k})}{\partial k_{x}} .
$$

To measure the Berry curvature $\Omega_{y}$, one need to collect the IF shift in $z$ direction $\Delta_{I F}(E, \theta)$ as a function of energy $E$ and incidence angle $\theta$. The $\Delta_{I F}(E, \theta)$ can be transformed into $\Delta_{I F}\left(k_{x}, k_{y}\right)$, and its derivative gives out the Berry curvature $\Omega_{y}(\mathbf{k})$. In the same way, the IF shift in other directions can be used to obtain $\Omega_{x}(\mathbf{k})$ and $\Omega_{z}(\mathbf{k})$.

Summary. - We obtained analytical expressions of the $\mathrm{GH}$ shift and IF shift at the interface of WSMs. We demonstrate that the IF shift is valley-dependent, and can be attributed to the topological nature of the system. This IF shift can lead to valley-dependent anomalous velocity, which is experimentally detectable. Finally, we discuss three applications of the topological IF shift including characterization of WSMs, fabrication of high efficient valleytronic devices, and detection the Berry curvature.

Acknowledgments.-This work was financially supported by NBRP of China (2012CB921303, 2012CB821402, and 2014CB920901) and NSF-China under Grants Nos. 11274364, and 91221302, and 11374219.
* These authors contributed equally to this work.

$\dagger$ sunqf@pku.edu.cn

$¥$ xcxie@pku.edu.cn

1 F. Goos, and H. Hächen, Ann. Phys. 1, 333 (1947).

2 F. Bretenaker, A. Floch, and L. Dutriaux, Phys. Rev. Lett. 68, 931 (1992).

3 M. Peccianti, A. Dyadyusha, M. Kaczmarek, and G. Assanto, Nature Physics 2, 737-742 (2006).

${ }^{4}$ F. I. K. Fedorov, Dokl. Akad. Nauk SSSR 105, 465-468
(1955).

${ }^{5}$ C. Imbert, Phys. Rev. D 5, 787 (1972).

6 M. Onda, S. Murakami, and N. Nagaosa, Phys. Rev. Lett. 93, 083901 (2004).

${ }^{7}$ K. Y. Bliokh and Y. P. Bliokh, Phys. Rev. Lett. 96, 073903 (2006).

8 O. Hosten and P. Kwiat, Science 319, 787-790 (2008).

9 X.Yin, Z. Ye, J. Rho, Y. Wang, and X. Zhang, Science 339, 1405-1407 (2013). 
10 S. C., Miller, Jr. and N. Ashby, Phys. Rev. Lett. 29, 740 (1972).

11 D. M. Fradkin and R. J. Kashuba, Phys. Rev. D 9, 2775 (1974).

12 X. Chen, X.-J. Lu, Y. Ban, and C.-F. Li, J. Opt. 15, 033001(2013).

13 V.-O. de Haan, J. Plomp, T. M. Rekveldt, W. H. Kraan, and Ad A. van Well, Phys. Rev. Lett. 104, 010401 (2010).

14 J. Huang, Z. Duan, H. Y. Ling, and W. Zhang, Phys. Rev. A 77, 063608 (2008).

15 C. W. J. Beenakker, R. A. Sepkhanov, A. R. Akhmerov, and J. Tworzydło, Phys. Rev. Lett. 102, 146804 (2009).

16 X. Wan, A. M. Turner, A. Vishwanath, and S. Y. Savrasov, Phys. Rev. B 83, 205101 (2011).

17 G. Xu, H. Weng, Z. Wang, X. Dai, and Z. Fang, Phys. Rev. Lett. 107, 186806 (2011).

18 A. A. Burkov and L. Balents, Phys. Rev. Lett. 107, 127205 (2011).

19 P. Hosur, S. A. Parameswaran, and A. Vishwanath, Phys. Rev. Lett. 108, 046602 (2012).

20 J.-H. Jiang, Phys. Rev. A 85, 033640 (2012).

${ }^{21}$ L. Lu, L. Fu, J. D. Joannopoulos, and M. Soljačić, Nature Photonics 7, 294-299 (2013).

22 S. A. Yang, H. Pan, and F. Zhang, Phys. Rev. Lett. 113, 046401 (2014).

23 H.B. Nielsen, M. Ninomiya, Nucl. Phys. B 185, 20-40 (1981).

24 H.B. Nielsen, M. Ninomiya, Phys. Lett. B 130, 389 (1983).

25 D. Yanagishima and Y. Maeno, J. Phys. Soc. Jpn. 70, 2880-2883 (2001).

26 S. T. Bramwell and M.J.P. Gingras Science 294, 1495-1501 (2001).

27 P. Hosur and X.-L. Qi Comptes Rendus Physique 14, 857 (2013).

28 A, Rycerz, J. Tworzydło, and C.W.J. Beenakker, Nature Physics 3, 172-175 (2007).

29 D. Xiao, W. Yao, and Q. Niu, Phys. Rev. Lett. 99: 236809 (2007).

30 K.F. Mak, K.L. McGill, J. Park, P.L. McEuen, Science 344, 1489-1492 (2014).

${ }^{31}$ R. V. Gorbachev, J. C. W. Song, G. L. Yu, A. V. Kretinin, F. Withers, Y. Cao, A. Mishchenko, I. V. Grigorieva, K. S. Novoselov, L. S. Levitov, A. K. Geim Science 346: 448-451 (2014).

32 See the Supplemental Materials for details.

33 Z. Fang, N. Nagaosa, K. S. Takahashi, A. Asamitsu, R. Mathieu, T. Ogasawara, H. Yamada, M. Kawasaki, Y. Tokura, K. Terakura, Science 302, 92-95 (2003).

${ }^{34}$ D. Xiao, M.-C. Chang, and Q. Niu, Rev. Mod. Phys. 82, 109 (2010).

35 M.-C. Chang and Q. Niu, Phys. Rev. B 53, 7010 (1996).

${ }^{36}$ Note that the velocity parameters are closely related to the hopping parameters, which can be tuned by strain on the bulk materials. See reference [37] for details.

37 Z. Wang, H. Weng, Q. Wu, X. Dai, and Z. Fang, Phys. Rev. B 88, 125427 (2013).

38 V. Aji, Phys. Rev. B 85, 241101 (2012).

39 D. T. Son and B.Z. Spivak, Phys. Rev. B 88, 104412 (2013).

40 S. A. Parameswaran, T. Grover, D.A. Abanin, D. A. Pesin, A. Vishwanath, Phys. Rev. X 4, 031035 (2014). 


\title{
Appendix A: Supplementary Information for "Topological Imbert-Fedorov shift in Weyl semimetals"
}

\author{
Qing-Dong Jiang, Hua Jiang, Haiwen Liu, Qing-feng Sun, and X. C. Xie
}

\section{Detail derivation of the GH and IF shifts.}

To calculate the spatial shifts of the wave packet in $y, z$ directions, one need to know the central positions of the incident and the reflected wave packets at the interface $(x=0)$. By expanding the phases $\alpha\left(k_{y}, k_{z}\right)$ and $\phi_{r}\left(k_{y}, k_{z}\right)$ to the first order around $\left(\bar{k}_{y}, \bar{k}_{z}\right)$, the integrals of $\psi_{g}^{i n}$ and $\psi_{g}^{r e}$ give that $\psi_{g \pm}^{i n} \propto e^{-\left(y \mp \frac{1}{2} \frac{\partial \alpha}{\partial k_{y}}\right)^{2} \Delta_{k_{y}}^{2} / 2} e^{-\left(z \mp \frac{1}{2} \frac{\partial \alpha}{\partial k_{z}}\right)^{2} \Delta_{k_{z}}^{2} / 2}$ and $\psi_{g \pm}^{r e} \propto e^{-\left(y+\frac{\partial \phi_{r}}{\partial k_{y}} \pm \frac{1}{2} \frac{\partial \alpha}{\partial k_{y}}\right)^{2} \Delta_{k_{y}}^{2} / 2} e^{-\left(z+\frac{\partial \phi_{r}}{\partial k_{z}} \pm \frac{1}{2} \frac{\partial \alpha}{\partial k_{z}}\right)^{2} \Delta_{k_{z}}^{2} / 2}$, where the \pm subscript corresponds to the first and the second component of the spinor, respectively. Then one can identify the center (maximum) of the incident and reflected wave packets in real space. For the incident wave packet, the two spinor components are centered at $\left(\bar{y}_{ \pm}^{i n}, \bar{z}_{ \pm}^{i n}\right)$, where

$$
\left\{\begin{array}{l}
\bar{y}_{ \pm}^{i n}=\left[ \pm \frac{1}{2} \frac{\partial}{\partial k_{y}} \alpha\left(k_{y}, k_{z}\right)\right]_{k_{y}=\bar{k}_{y}, k_{z}=\bar{k}_{z}} \\
\bar{z}_{ \pm}^{i n}=\left[ \pm \frac{1}{2} \frac{\partial}{\partial k_{z}} \alpha\left(k_{y}, k_{z}\right)\right]_{k_{y}=\bar{k}_{y}, k_{z}=\bar{k}_{z}}
\end{array}\right.
$$

For the reflected wave packet, the two spinor components are centered at $\left(\bar{y}_{ \pm}^{r e}, \bar{z}_{ \pm}^{r e}\right)$, where

$$
\left\{\begin{array}{l}
\bar{y}_{ \pm}^{r e}=\left[-\frac{\partial}{\partial k_{y}} \phi_{r}\left(k_{y}, k_{z}\right) \mp \frac{1}{2} \frac{\partial}{\partial k_{y}} \alpha\left(k_{y}, k_{z}\right)\right]_{k_{y}=\bar{k}_{y}, k_{z}=\bar{k}_{z}} . \\
\bar{z}_{ \pm}^{r e}=\left[-\frac{\partial}{\partial k_{z}} \phi_{r}\left(k_{y}, k_{z}\right) \mp \frac{1}{2} \frac{\partial}{\partial k_{z}} \alpha\left(k_{y}, k_{z}\right)\right]_{k_{y}=\bar{k}_{y}, k_{z}=\bar{k}_{z}}
\end{array}\right.
$$

Considering above results, we can obtain the spatial shifts for two spinor components in $y, z$ directions, which are

$$
\left\{\begin{array}{c}
\Delta_{ \pm}^{y}=\bar{y}_{ \pm}^{r e}-\bar{y}_{ \pm}^{i n}=-\frac{\partial}{\partial k_{y}} \phi_{r}\left(\bar{k}_{y}, \bar{k}_{z}\right) \mp \frac{\partial}{\partial k_{y}} \alpha\left(\bar{k}_{y}, \bar{k}_{z}\right) \\
\Delta_{ \pm}^{z}=\bar{z}_{ \pm}^{r e}-\bar{z}_{ \pm}^{i n}=-\frac{\partial}{\partial k_{z}} \phi_{r}\left(\bar{k}_{y}, \bar{k}_{z}\right) \mp \frac{\partial}{\partial k_{z}} \alpha\left(\bar{k}_{y}, \bar{k}_{z}\right)
\end{array}\right.
$$

As is shown in Eq. A3), in order to calculate the spatial shift at the interface $(\mathrm{x}=0)$, we need to know the reflection phase $\phi_{r}\left(\bar{k}_{y}, \bar{k}_{z}\right)$ and $\alpha\left(k_{y}, \bar{k}_{z}\right) . \alpha\left(\bar{k}_{y}, \bar{k}_{z}\right)$ represents the incident angle of the wave packet, and can be easily obtained once we know the incident wave vector. Next, we elaborate on deriving the reflection phase $\phi_{r}$. We consider the total reflection case, i.e., the reflection probability $|r|^{2}=1$. Thus, the wave function must be evanescent in the region $x>0\left[\right.$ Fig. 2(a)]. We can calculate the reflection coefficient by matching the wave function $\psi^{\text {in }}(\mathbf{k}, \mathbf{r})+\psi^{r e}(\mathbf{k}, \mathbf{r})$ at $x=0$ to the evanescent wave. The continuity of wave function gives the reflection amplitude $r=e^{i \phi_{r}}$, where $\phi_{r}=$ $2 \tan ^{-1}\left(\frac{\eta \cos \alpha}{-\eta \sin \alpha+\xi}\right)-\alpha-\pi / 2$. In this expression, $\xi=\frac{\hbar\left(\kappa+k_{y}\right)}{E-V+\hbar v_{z}^{\prime} k_{z}}$, where $\kappa=\sqrt{\left(\hbar v_{y}^{\prime} k_{y}\right)^{2}+\left(\hbar v_{z}^{\prime} k_{z}\right)^{2}-(E-V)^{2}} / \hbar v_{x}^{\prime}$. Substitute the expression of $\alpha$ and $\phi_{r}$ into Eq.A3, and we can obtain the average shifts in $y, z$ directions.

\section{IF shift for nonlinear energy dispersion.}

In the main text, we have shown that the Berry curvature is the origin of the IF shift for WSMs, which possess linear energy band dispersions. Here, we show that the Berry curvature is still the origin of the IF shift for another system without linear energy dispersions. To clarify this idea, we consider a system with a model Hamiltonian

$$
\mathcal{H}^{\prime}=\left(\begin{array}{cc}
A k_{y}^{2} & B\left(k_{x}-i k_{z}\right) \\
B\left(k_{x}+i k_{z}\right) & -A k_{y}^{2}
\end{array}\right)
$$

where $A$ and $B$ are two parameters. The eigenenergy of the Hamiltonian is $E= \pm \sqrt{B^{2}\left(k_{x}^{2}+k_{z}^{2}\right)+A^{2} k_{y}^{4}}$. Thus this Hamiltonian obviously does not have 3D linearized energy dispersions. One can apply a gate voltage $V$ to this system at the region $x \geqslant 0$; therefore, an interface appears at $x=0$.

We now consider that a wave packet incidents on the interface from the region $x<0$. To calculate the IF shift at the interface, we follow the same procedure in the main text. Analogously, we first construct the incident wave packet in Gaussian profile with center momentum $\left(\bar{k}_{x}, \bar{k}_{y}, \bar{k}_{z}\right)$. Still we assume the wave packet as $\psi_{g}^{\text {in }}(\mathbf{r})=$ $\int_{-\infty}^{\infty} \int_{-\infty}^{\infty} d k_{y} d k_{z} f\left(k_{y}-\bar{k}_{y}\right) f\left(k_{z}-\bar{k}_{z}\right) \psi^{i n}(\mathbf{k}, \mathbf{r})$, where $f\left(k_{s}-\bar{k}_{s}\right)$ has the same definition as in the main text. Similarly, the reflected wave packet can be constructed as $\psi_{g}^{r e}(\mathbf{r})=\int_{-\infty}^{\infty} \int_{-\infty}^{\infty} d k_{y} d k_{z} f\left(k_{y}-\bar{k}_{y}\right) f\left(k_{z}-\bar{k}_{z}\right) \psi^{r e}(\mathbf{k}, \mathbf{r})$. However, 
since the Hamiltonian $\mathcal{H}^{\prime}$ here is different from that in the main text, one need to solve the wave functions $\psi^{\text {in }}(\mathbf{k}, \mathbf{r})$ and $\psi^{r e}(\mathbf{k}, \mathbf{r})$ for $\mathcal{H}^{\prime}$. The Hamiltonian equation is $\mathcal{H}^{\prime} \psi=E \psi$, where $\psi=\left(\psi_{1}, \psi_{2}\right)^{\mathrm{T}}$ is a two components spinor. Solve this Hamiltonian equation and use continuity condition of the wave function at $x=0$, then we can get the solutions:

$$
\psi^{i n}(\mathbf{k}, \mathbf{r})=\frac{1}{\sqrt{1+\zeta^{2}}}\left(\begin{array}{c}
e^{-i \alpha\left(k_{y}, k_{z}\right) / 2} \\
\zeta e^{i \alpha\left(k_{y}, k_{z}\right) / 2}
\end{array}\right) e^{i k_{x} x+i k_{y} y+i k_{z} z},
$$

and

$$
\psi^{r e}(\mathbf{k}, \mathbf{r})=\frac{r}{\sqrt{1+\zeta^{2}}}\left(\begin{array}{c}
-i e^{i \alpha\left(k_{y}, k_{z}\right) / 2} \\
i \zeta e^{-i \alpha\left(k_{y}, k_{z}\right) / 2}
\end{array}\right) e^{-i k_{x} x+i k_{y} y+i k_{z} z}
$$

In the above expressions, $k_{x}=\sqrt{E^{2}-\left(A k_{y}^{2}\right)^{2}-\left(B k_{z}\right)^{2}} / B, \alpha=\tan ^{-1}\left(k_{z} / k_{x}\right), \zeta=\sqrt{\frac{E-\alpha k_{y}^{2}}{E+\alpha k_{y}^{2}}}, r=|r| e^{i \phi_{r}}$ is the reflection amplitude, and $\phi_{r}=2 \tan ^{-1}\left(\frac{\zeta \cos \theta}{-\zeta \sin \theta+\xi^{\prime}}\right)$ with $\xi^{\prime}=\frac{B\left(\kappa+k_{z}\right)}{E-V+A k_{y}^{2}}$ and $\kappa=\sqrt{(E-V)^{2}-\left(A k_{y}^{2}\right)^{2}-\left(B k_{z}\right)^{2}} / B$. Substitute $\phi_{r}$ and $\alpha$ into Eq. $\sqrt{\mathrm{A} 3}$, and one can obtain the spatial shifts in $y$ and $z$-directions. If the incident wave packet is confined in $x-y$ plane, then the out-of-plane spatial shift in $z$-direction corresponds to the IF shift and reads:

$$
\Delta_{I F}=\frac{B^{2}}{A} \frac{k_{x}}{E k_{y}^{2}}
$$

Alternatively, one can also obtain the IF shift $\Delta_{I F}$ from semiclassical equation for wave packet (Eq.(7) in the main text). In order to apply the semiclassical equation, one need calculate the Berry curvature $\boldsymbol{\Omega}^{ \pm}$for the Hamiltonian $\mathcal{H}^{\prime}$, where the superscript \pm corresponds to the conductance band and valence band, respectively. The Berry curvature of this system is $\Omega^{ \pm}=\left(\mp \frac{A B^{2}}{2 E^{3}} k_{x} k_{y}, \mp \frac{A B^{2}}{2 E^{3}} k_{y}^{2}, \mp \frac{A B^{2}}{2 E^{3}} k_{z} k_{y}\right)$. Substitute the Berry curvature expression into the Eq.(11) in the main text, and one can obtain the analytic result of the IF shift $\Delta_{I F}=\frac{B^{2}}{A} \frac{k_{x}}{E k_{y}^{2}}$, showing the consistence with Eq. A7). The fact that the IF shift $\Delta_{I F}$ can be obtained from Eq.(7) in the main text provides another convincing argument that IF shift arises from the Berry curvature of the system.

\section{Calculations of anomalous velocities and position shifts for two valleys.}
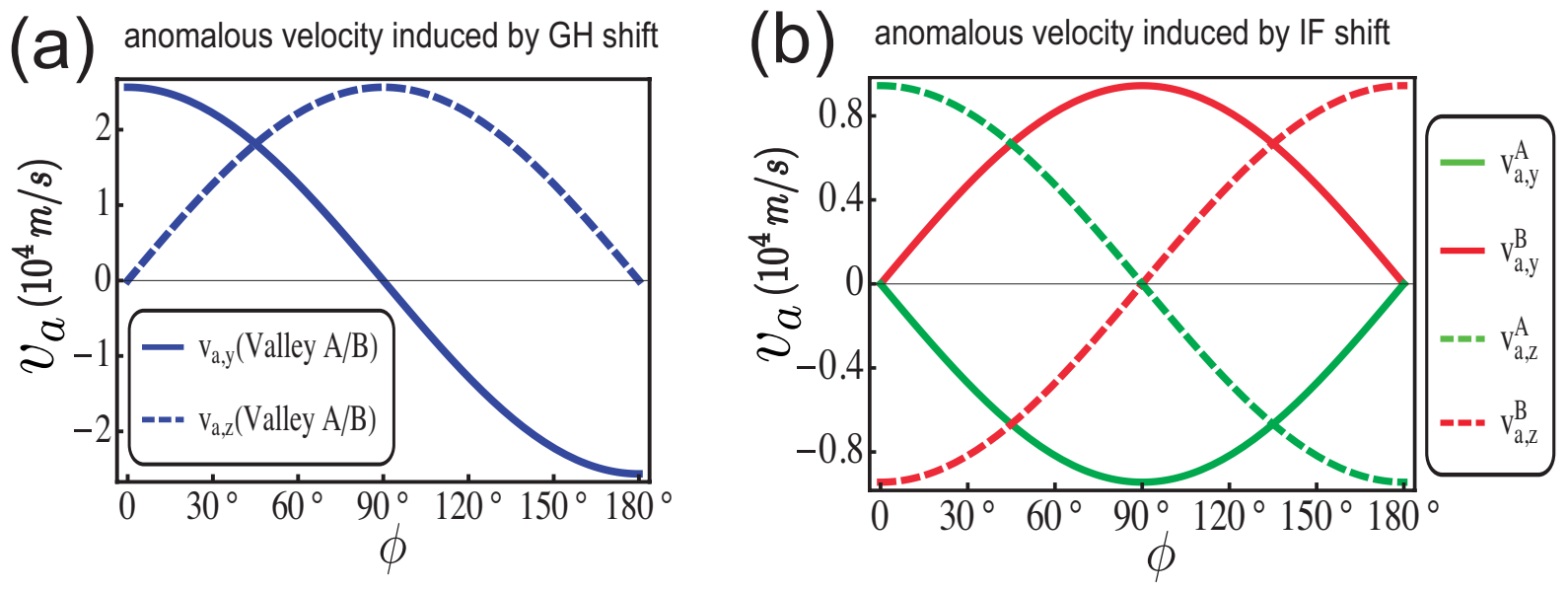

FIG. 5: (a) The anomalous velocities induced by the GH shift are valley-independent. (b)The anomalous velocities induced by the IF shift are valley-independent.

In the main text, we have demonstrated that the GH shift does not depend on valley index, whereas the IF shift depends on valley index. Although both of the GH shift and IF shift can bring anomalous velocities to the normal velocities, only the anomalous velocity induced by IF shift is valley-dependent. There are both GH and IF shifts at the left interface $\left(x=-\frac{d}{2}\right)$ and the right interface $\left(x=\frac{d}{2}\right)$. If the system has the mirror symmetry about the $x=0$ plane, the GH shifts are in the same direction at the left and right interfaces. But IF shifts are in opposite direction at 
these two interfaces, leading to the cancellation of the IF shifts after two subsequent reflections. Therefore, in order to observe the valley-dependent IF shift, the mirror symmetry about $x=0$ plane need to be broken. So we consider that the $\mathrm{z}$ direction velocities in the three regions are unequal, with $v_{z}^{\mathrm{I}}=v_{L}, v_{z}^{\mathrm{II}}=v$, and $v_{z}^{\mathrm{III}}=v_{R}$. For simplicity, the $\mathrm{x}$ and y directions velocities are still with $v_{x / y}^{\mathrm{I}}=v_{x / y}^{\mathrm{II}}=v_{x / y}^{\mathrm{III}}=v$. Figure $\mathrm{S} 1$ shows the anomalous velocities induced by the GH shift and IF shift. The anomalous velocities are calculated with the same parameters as in the section "Anomalous velocities induced by IF effect" in the main text. As one can see, the anomalous velocity induced by the GH shift is valley-independent; by contrast, the anomalous velocity induced by the IF shift is valley-dependent. The green curves and red curves correspond to valley $\mathrm{A}$ and valley $\mathrm{B}$, respectively. The thick curves and dashed curves correspond to the anomalous velocities in y-direction and z-direction, respectively.

Given the incident direction $(\theta, \phi)$ of the incident wave packet, we can calculate the final position $(x, y, z)$ at bottom of the region II. The wave packet needs time $T_{0}=h / v_{n, z}$ to reach to the bottom of the region II without considering anomalous velocity. However, if we take the anomalous velocity into account, the wave packet needs time $T_{1}=h / v_{t, z}$ to reach to the bottom of the region II. Thus the anomalous velocities induced position shift is

$$
\Delta L=v_{t, y} \times T_{1}-v_{n, y} \times T_{0} .
$$

Put the total velocities of the valley A and valley B into Eq. A8, we can get the final position shifts for the valley A and valley $\mathrm{B}$, respectively [see Fig. 3(d) in the main text].

\section{Calculations of valley density distribution induced by valley-dependent anomalous velocities.}
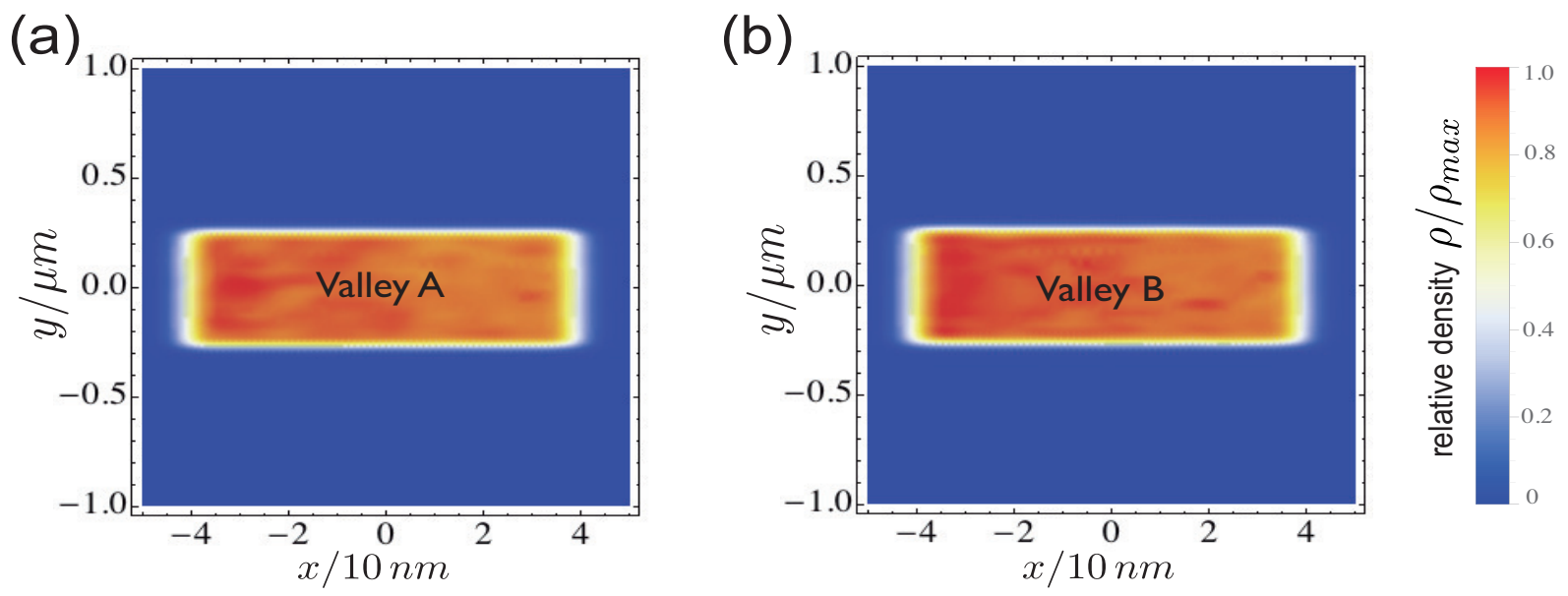

FIG. 6: Valley pattern before considering the IF shift. The figures (a) and (b) show the valley pattern of valley A and B, respectively, before considering the anomalous velocities induced by the spatial shifts. The color bar on the right represents the relative density of Weyl particles. Here, the relative density has the same meaning as that in the main text.

Since the incident wave packets are likely to be reflected repeatedly at two interfaces, one only needs to consider the total reflected cases since the partially reflected wave packets would eventually diminish after being reflected multiple times. A collimated incident wave packet usually has certain angle range, thus, we assume $\theta \in\left[\theta_{c}-\delta / 2, \theta_{c}+\delta / 2\right]$ and $\phi \in\left[\phi_{c}-\delta / 2, \phi_{c}+\delta / 2\right]$, where $\theta_{c}$ and $\phi_{c}$ are the angles of the center of the collimated incident wave packet and the $\delta$ is the angle spread range. In the numerical calculations, we take $\theta_{c}=32.5^{\circ}$ and $\phi_{c}=90^{\circ}$. In this case, the anomalous velocity $v_{a, y}$ induced by the GH effect is negative, and the anomalous velocity induced by the IF shift is relatively large. Hence, the total anomalous velocity has a remarkable valley-dependent effect.

For a wave packet of Weyl fermions propagating in the Region II, the total velocities in $y, z$ directions are $v_{t, y}$ and $v_{t, z}$, respectively. Due to the valley-dependent anomalous velocities, the total velocities are also valley-dependent, which can lead to macroscopic shifts of valleys. Because of the reflection at the two interfaces (Fig. 3(B)), the velocity in x direction $v_{x}^{I I}$ in the Region II is a periodic function of $\Delta t$, i.e., $v_{x}^{I I}(t)=v_{x}^{I I}(t+2 \Delta t)$. In the first period, the velocity $v_{x}^{I I}(t)$ reads

$$
v_{x}^{I I}(t)=\left\{\begin{array}{cc}
v_{x} & t \in\left[0, \frac{\Delta t}{2}\right) \cup\left[\frac{3 \Delta t}{2}, 2 \Delta t\right] \\
-v_{x} & t \in\left[\frac{\Delta t}{2}, \frac{3 \Delta t}{2}\right)
\end{array}\right.
$$


The height of this structure is $h$, thus the wave packet needs time $t_{0}=h / v_{t, z}$ to reach to the bottom of the Region II. Hence, one can get the final position coordinate $(x, y, z)$ of the wave packet, where $x=\int_{0}^{t_{0}} d t v_{x}^{I I}(t), y=\int_{0}^{t_{0}} d t v_{t, y}$, and $z=-h$. As one can see, a single incident direction $(\theta, \phi)$ of the wave packet corresponds to a single point $(x, y, z)$ at the bottom of Region II. We further assume that the injector supplies an equal strength of incident beams in the angle range $\theta \in\left[32.5^{\circ}-\delta / 2,32.5^{\circ}+\delta / 2\right]$ and $\phi \in\left[90^{\circ}-\delta / 2,90^{\circ}+\delta / 2\right]$. Thus, there will be a pattern (density distribution) at the bottom of Region II [see Fig. 4(d) in the main text]. The Figure S2 (a) and (b) show the density distribution of valley A and valley B, respectively, before considering the IF shift. From the Figure S1(a) and (b) we can find that the density distribution of valley A and valley B are located at the same place. Therefore, the Weyl fermions from valley A and valley B are mixed together. However, if the anomalous velocity is taken into account, the distribution of valley A and valley B will shift to opposite direction leading to the valley separation [as shown in Fig. 4(a), (b) in the main text].

\section{Supplementary References}

* These authors contributed equally to this work.

† sunqf@pku.edu.cn

¥ xcxie@pku.edu.cn

1 D. Xiao, M.-C. Chang, and Q. Niu, Rev. Mod. Phys. 82, 109 (2010). 